\title{
Anestesia em cães utilizando éter glicerol guaiacol, quetamina e fentanil em infusão contínua intravenosa
}

\section{Anaesthesia in dogs using continuous intravenous infusion of guaiphenesin, ketamine and fentanyl}

\author{
Fernando Augusto Ferreira Vieira, Stélio Pacca Loreiro Luna, Mariangela Losano Cruz, Gladys Bastos de Castro, \\ Flávio Massone
}

\section{Resumo}

Onze cães, sem raça definida, receberam como medicação pré-anestésica a anestesia induzida e mantida por infusão contínua intravenosa de quetamina e fentanil em solução de éter gliceril guaiacol a $5 \%$ em glicose a $5 \%$, através de bomba de infusão. Em seis animais foram mensuradas freqüência cardíaca e respiratória, ventilometria (volume corrente e volume minuto), pressão arterial (sistólica, média e diastólica), temperatura retal, hemogasometria arterial, saturação de $\mathrm{O}_{2}$ na hemogoblina, anion residual, osmolalidade sérica, concentração sérica total de $\mathrm{O}_{2}$, hematócrito, hemoglobina, glicose e eletrólitos. Avaliou-se também a analgesia através de pinçamento da pele da região glabra. Em outro grupo de cinco animais, onde foram realizadas três ovários-salpingo-histerectomias e duas osteossínteses, foram mensuradas a freqüência cardíaca e respiratória e a resposta ao estímulo cirúrgico. Em ambos os grupos observou-se, principalmente, depressão respiratória dose-dependente, agravada com o uso de velocidade de infusão elevada, no caso de manobras cirúrgicas muito cruentas, como manipulação de periósteo.

Palavras chave: éter gliceril guaiacol; quetamina; fentanil; infusão contínua intravenosa; cães

\section{Introdução}

A administração de anestésicos por via intravenosa em infusão contínua minimiza flutuações na concentração plasmática dos mesmos, diminuindo o risco de sobredoses ou subdoses e proporcionando maior estabilidade cardiovascular; quando a velocidade de infusão é adequada, a recuperação anestésica é rápida, acompanhada de analgesia e isenta de excitação, reduzindo-se ainda a incidência de efeitos colaterais (Miller, 1994). O consumo de anestésicos é diminuído quando os mesmos são administrados pela referida técnica, em relação ao uso de bolus sucessivos (White, 1983).
É bem documentado o uso do éter glicerol guaiacol (EGG) em cães por infusão contínua intravenosa em associação a outras drogas (Benson et al., 1985a; Benson et al., 1985b; Ratajczak e Skyrzypczak, 1993). Entretanto, todos os protocolos citados, incluem a associação de xilazina e quetamina, observando-se diminuição do débito cardíaco por redução no volume de ejeção, fato imputável à dogra $\mathrm{a}_{2}$-agonista (Benson et al., 1985a).

$O$ objetivo deste trabalho foi substituir a droga $a_{2}-$ agonista na associação com quetamina e EGG por um opióide agonista, o fentanil, com o intuito de se obter maior estabilidade dos parâmetros cardiovasculares.

\section{Material e Métodos}

Foram utilizados onze cães, sem raça definida, seis (G1) oriundos do Biotério Central e cinco (G2) da rotina de atendimento do Hospital Veterinário da FMVZ-UNESP, Botucatu, Estado de São Paulo, com peso de $15.1 \pm 6.5$ $\mathrm{kg}$, e idade de $33.8 \pm 23$ meses, clinicamente sadios, submetidos a jejum alimentar de $12 \mathrm{~h}$ e hídrico de $2 \mathrm{~h}$ antes do experimento.

Após aplicação da medicação pré-anestésica (MPA) a base de levomepromazina ${ }^{1}(1.0 \mathrm{mg} / \mathrm{kg})$ por via intravenosa, a anestesia foi induzida com solução contendo $\mathrm{EGG}^{2}$ a $5 \%$ em glicose a $5 \%{ }^{3}$, quetamina ${ }^{4}(2.0 \mathrm{mg} /$ $\mathrm{ml})$ e fentanil ${ }^{5}(0.005 \mathrm{mg} / \mathrm{ml})$, na dose de $1 \mathrm{ml} / \mathrm{kg}$ por via intravenosa, em injeção lenta (por $180 \mathrm{seg}$.). A anestesia foi mantida por infusão contínua intravenosa de solução contendo EGG a 5\% em glicose a 5\%, quetamina (4 mg/ $\mathrm{ml}$ ) e fentanil $(0.015 \mathrm{mg} / \mathrm{ml})$, através de bomba de infusão por gravidade com controle elétrico de gotejamento ${ }^{6}$, em todos os animais.

Em seis animais (G1) foram mensuradas freqüência cardíaca e respiratória, ventilometria (volume corrente e volume minuto), pressão arterial (sistólica, média e diastólica), temperatura retal, hemogasometria arterial $\left(\mathrm{pH}, \mathrm{PaO}_{2}, \mathrm{CO}_{2}\right.$ total, bicarbonato e excesso de base), saturação de $\mathrm{O}_{2}$ na hemoglobina, anion residual,

\footnotetext{
Departamento de Cirurgia Veterinária e Reprodução Animal - FMVZ-UNESP, 18603-170 Botucatu, SP, Brasil

${ }^{1}$ Neozine, Rhodia

2 Éter Gliceril Guaicacol, Henrifarma

${ }^{3}$ Glicose a 5\%, Labormédica
} 
osmolalidade sérica, concentração sérica total de $\mathrm{O}_{2}$, hematócrito, hemoglobina, glicose e eletrólitos $\left(\mathrm{Na}^{+}, \mathrm{K}^{+}\right.$, $\mathrm{Cl}, \mathrm{Ca}^{++}$e cálcio não ionizado) séricos. A analgesia foi testada através do pinçamento da pele da região glabra e do espaço interdigital. Sangue arterial foi colhido anaerobiamente, por meio de punção de uma das artérias femorais dos cães, usando-se seringas plásticas e agulhas hipodérmicas $30 \times 6$, após tricotomia e antissepsia da região correspondente; as amostras acondicionadas em recicipente isotérmico a $4^{\circ} \mathrm{C}$. A freqüência cardíaca e a pressão arterial foram mensuradas através de manômetro digital não invasivo ${ }^{7}$, a freqüência respiratória e a ventilometria por meio de ventilômetro ${ }^{8}$ e a temperatura com termômetro clínico digital ${ }^{9}$. Todas as análises séricas foram realizadas em aparelho de hemogasometria ${ }^{10}$. As freqüências cardíaca e respiratória, pressão arterial, temperatura retal e ventilometria foram registradas antes da MPA e aos $5,10,20,30,40,50$ e 60 min. após a indução anestésica. O sangue arterial para as análises supracitadas foi coletado antes da MPA e aos 5, 30 e 60 min. após a indução anestésica, e aos 15 min. após o fim da infusão da solúção de manutenção. Neste grupo, a infusão da solução de manutenção foi mantida por 60 min. após a indução anestésica.

Em cinco animais (G2) as freqüências cardíaca e respiratória foram mensuradas, por meio de estetoscópio esofágico ${ }^{11}$, antes da MPA e a cada $5 \mathrm{~min}$. até o fim da infusão da solução de manutenção, com duração variando de acordo com o tempo cirúrgico. Nestes animais foram realizadas três ovário-salpingo-histerectomias e duas osteossínteses; objetivou-se, tão somente, a avaliação do paciente face a estímulos cirúrgicos diversos, não sendo os animais utilizados para fins de análise estatística.

Todos os cães foram intubados por via orotraqueal com sonda de Magil'12, imediatamente após a indução anestésica. Em G1, os animais respiraram ar ambiente e em G2 o traqueotubo era conectado a um circuito anestésico, administrando-se $\mathrm{O}_{2}$ puro ao paciente. A qualidade de recuperação foi avaliada pela mesma pessoa até que todos os animais assumissem a posição de estação.

\section{Análise estatística}

A análise estatística foi realizada de acordo com Curi (1980), utilizando-se o programa Staview em computador Mackintosh. Para avaliação de diferença dentro de cada grupo, compararam-se valores paramétricos observados após a indução anestésica com o momento anterior à aplicação da medicação pré-anestésica, utilizan- do-se análise de variância (ANOVA) para amostras repetidas, seguida pelo teste de Dunnett (Dunnett, 1964) quando necessário. Diferenças foram consideradas significativas quando $p<0.05$. As tabelas estão expressas em média \pm desvio padrão.

\section{Resultados}

A freqüência cardíaca, a pressão arterial sistólica, a diastólica e a média não apresentaram alterações significativas (Tabela 1).

Nos cães (G1) a freqüência respiratória e o volume minuto decresceram significativamente após a indução e durante toda a manutenção anestésica, com redução significativa do volume corrente somente aos $5 \mathrm{~min}$. após a indução anestésica. $\mathrm{A} \mathrm{PaO}_{2}$ reduziu significativamente dos 5 aos 60 min. após a indução anestésica, retornando aos níveis de controle $15 \mathrm{~min}$. após o fim da infusão. A acidose respiratória foi observada dos 5 aos $75 \mathrm{~min}$. após a indução anestésica. Observou-se aumento significativo dos níveis séricos de bicarbonato e $\mathrm{CO}_{2}$ total dos 5 aos $60 \mathrm{~min}$. após a indução. Os valores de saturação de $\mathrm{O}_{2}$, de concentração sérica total de $\mathrm{O}_{2}$ e de excesso de base não apresentaram alterações significativas durante a anestesia (Tabelas 1 e 2).

Hipotermia foi observada dos 10 aos $60 \mathrm{~min}$. após a indução anestésica (Tabela 1).

Não houve alteração significativa nos valores de hematócrito, hemoglobina, osmolalidade, anion residual, glicose e eletrólitos, excetuando-se redução na concentração sérica de potássio, aos 60 e 75 min. após a indução anestésica (Tabela 3).

Nos animais (G1) foram infundidos $1.56 \mathrm{ml} / \mathrm{kg} / \mathrm{h}$ da solução de manutenção durante uma hora, administrando-se assim aos animais $78 \mathrm{mg} / \mathrm{kg} / \mathrm{h}$ de EGG, $6.24 \mathrm{mg} / \mathrm{kg} / \mathrm{h}$ de quetamina e $0.023 \mathrm{mg} / \mathrm{kg} / \mathrm{h}$ ora de fentanil. Foram extubados, respectivamente, aos $12 \pm 1.07 \mathrm{~min}$., movimentaram a cabeça aos $40.5 \pm 14.1$ min., assumiram decúbito esternal aos $60.17 \pm 15.5$ e posição de estação aos 75.17 $\pm 21.6 \mathrm{~min}$. após o término da infusão de manutenção enquanto que nos animais (G2) cada evento ocorreu respectivamente aos $4.5 \pm 1.3,36.25 \pm 10.1,56 \pm 12.4$ e $83.3 \pm$ 17.9 min após o término da infusão anestésica.

Um animal (G2), de $6.3 \mathrm{~kg}$ e idade de 12 meses, submetido a osteossíntese umeral apresentou resposta ventilatória (taquipnéia com inspirações bruscas), hemodinâmica (taquicardia) e motora (tremor lingual) à manipulação de periósteo. A estabilização paramétrica foi obtida mediante o aumento da velocidade de infusão, com conseqüente apnéia; foi instituída a ventilação controlada manual por pressão positiva intermitente até o fim do ato

\footnotetext{
${ }^{7}$ Manômetro digital não invasivo DX 710, Dixtal

${ }^{8}$ Ventilômetro de Wright - Volumeter 3000 , Drager

${ }^{9}$ Termômetro clínico digital MT-500-1, Microtherm
}

\footnotetext{
${ }^{10}$ Blood Gas Analyser - Stat Profile 5, Nova Biomedical

${ }^{11}$ Estetoscópio Esofágico, Biotecno

${ }^{12}$ Rüsch
} 
Tabela 1 - Freqüência cardíaca e respiratória, volume corrente, pressão arterial sistólica, diastólica e média e temperatura retal de cães anestesiados com éter glicerol guaiacol, quetamina e fentanil $(n=6)$

\begin{tabular}{lcccccccc}
\hline \multicolumn{1}{c}{} & 0 & $5^{\prime}$ & $10^{\prime}$ & $20^{\prime}$ & $30^{\prime}$ & $40^{\prime}$ & $50^{\prime}$ & $60^{\prime}$ \\
\hline Freqüência & & & & & & & & \\
cardíaca (bpm) & $104 \pm 9.5$ & $115 \pm 23.3$ & $120 \pm 27.1$ & $119 \pm 26.2$ & $119 \pm 30.1$ & $114 \pm 27.5$ & $112 \pm 25.4$ & $112 \pm 21.6$ \\
FR $(\mathrm{mrm})$ & $41 \pm 31.1$ & $16 \pm 5.2^{*}$ & $14 \pm 5.5^{*}$ & $13 \pm 4.6^{*}$ & $13 \pm 5.1^{*}$ & $12 \pm 5.5^{*}$ & $13 \pm 5.4^{*}$ & $13 \pm 5.6^{*}$ \\
VC $(\mathrm{ml} / \mathrm{kg})$ & $13.27 \pm 5.74$ & $8.03 \pm 2.63^{*}$ & $8.4 \pm 2.13$ & $8.78 \pm 2.02$ & $9.68 \pm 2.48$ & $9.02 \pm 2.26$ & $9.12 \pm 2.15$ & $8.74 \pm 2.28$ \\
VM $(\mathrm{l} / \mathrm{mim})$ & $7.1 \pm 3.2$ & $2.0 \pm 1.2^{*}$ & $1.9 \pm 1.2^{*}$ & $1.8 \pm 1.2^{*}$ & $2.1 \pm 1.7^{*}$ & $1.8 \pm 1.6^{*}$ & $2.1 \pm 1.5^{\star}$ & $1.9^{*} \pm 1.3^{*}$ \\
PAS $(\mathrm{mmHg})$ & $118 \pm 14.3$ & $116.2 \pm 18$ & $121.2 \pm 17.1$ & $116.9 \pm 17.3$ & $115 \pm 14.5$ & $113.4 \pm 10.3$ & $116.6 \pm 14.2$ & $117.6 \pm 9.5$ \\
PAM $(\mathrm{mmHg})$ & $93.2 \pm 21.1$ & $89.2 \pm 17$ & $82.5 \pm 14.1$ & $83.3 \pm 18.5$ & $82.5 \pm 18$ & $80.3 \pm 17.1$ & $88 \pm 15.8$ & $82.8 \pm 13.1$ \\
PAD $(\mathrm{mmHg})$ & $76.8 \pm 22$ & $73.7 \pm 17.3$ & $67.5 \pm 18.7$ & $66.8 \pm 22.5$ & $66 \pm 21.1$ & $62.8 \pm 19.2$ & $68.3 \pm 20.7$ & $67.8 \pm 16.6$ \\
TR $\left({ }^{\circ} \mathrm{C}\right)$ & $38.6 \pm 0.3$ & $37.7 \pm 0.8$ & $37.4 \pm 0,9^{*}$ & $37.2 \pm 1^{*}$ & $37.1 \pm 1^{*}$ & $36.6 \pm 0.3^{*}$ & $36.7 \pm 1.1$ & $36.5 \pm 1.1^{*}$ \\
\hline
\end{tabular}

0 - Momento imediatamente antes da aplicação de MPA

* - Valores significativamente diferentes dos observados antes da MPA

FC - Freqüência cardíaca (batimento/minuto)

FR - Freqüência respiratória (movimento respiratórios/minuto)

VC - Volume corrente (ml)

VM - Volume minuto (litros/minuto)

PAS - Pressão arterial sistólica

PAM - Pressão arterial média

PAD - Pressão arterial diastólica

TR - Temperatura retal

75' - 15 min. após o término da manutenção anestésica

Tabela 2 - Valores hemogasométricos em sangue arterial de cães anestesiados com éter glicerol guaiacol, quetamina e fentanil $(n=6)$

\begin{tabular}{lccccc}
\hline & 0 & $5^{\prime}$ & $30^{\prime}$ & 60 & $75^{\prime}$ \\
\hline $\mathrm{PaO}_{2}(\mathrm{mmHg})$ & $89.8 \pm 4.05$ & $65.7 \pm 15,68^{*}$ & $65.5 \pm 16.38^{*}$ & $65.1 \pm 17.81^{*}$ & $89 \pm 13.41$ \\
$\mathrm{PaCO}_{2}(\mathrm{mmHg})$ & $32.5 \pm 5.03$ & $45.4 \pm 5.39^{*}$ & $52.6 \pm 7.59^{*}$ & $57.4 \pm 7.4^{*}$ & $46.1 \pm 8.74^{\star}$ \\
$\mathrm{HCO}_{3}-(\mathrm{mmol} / \mathrm{l})$ & $21.1 \pm 3.97^{\star}$ & $23.8 \pm 3.97^{\star}$ & $24.7 \pm 3.72^{*}$ & $25.3 \pm 3.13^{*}$ & $23.3 \pm 3.41$ \\
$\mathrm{CO}_{2} \mathrm{~T}(\mathrm{mmol} / \mathrm{l})$ & $21.8 \pm 3.2$ & $25.2 \pm 4.1^{*}$ & $26.3 \pm 3.9^{*}$ & $27 \pm 3.06^{*}$ & $24.4 \pm 3.14$ \\
$\mathrm{~EB}(\mathrm{mmol} / \mathrm{l})$ & $-2.1 \pm 2.83$ & $-1.57 \pm 3.94$ & $-1.8 \pm 3.68$ & $-1.9 \pm 3.33$ & $-2.35 \pm 3.25$ \\
$\mathrm{O}_{2} \mathrm{SAT}(\%)$ & $97.1 \pm 0.53$ & $86.7 \pm 11.83$ & $85.8 \pm 11.04$ & $83.8 \pm 14.03$ & $95.2 \pm 2.64$ \\
$\mathrm{O}_{2} \mathrm{CT}(\mathrm{ml} / \mathrm{dl})$ & $13.1 \pm 0.68$ & $11.9 \pm 2.46$ & $12.2 \pm 3,97$ & $12,9 \pm 3.2$ & $14.5 \pm 4.3$ \\
$\mathrm{pH}$ & $7.42 \pm 0.03$ & $7.33 \pm 0.03^{*}$ & $7.28 \pm 0.04$ & $7.25 \pm 0.05^{\star}$ & $7.31 \pm 0.05^{*}$ \\
\hline
\end{tabular}

0 - Momento imediatamente antes da aplicação de MPA

* - Valores significativamente diferentes dos observadores antes da MPA

$\mathrm{PaO}_{2}$ - Tensão. de oxigênio arterial

$\mathrm{PaCO}_{2}$-Tensão de dióxido de carbono arterial

$\mathrm{HCO}_{3}$ - Concentração sérica arterial de bicarbonato

$\mathrm{CO}_{2} \mathrm{~T}$ - Concentração sérica arterial total de dióxido de carbono

EB - Excesso de base

$\mathrm{O}_{2}$ SAT-Saturação arterial de oxigênio na hemoglobina

$\mathrm{O}_{2} \mathrm{CT}$ - Concentração sérica arterial de oxigênio

$75^{\prime}$ - 15 min. após o término da manutenção anestésica

cirúrgico. Visto que o objetivo deste trabalho foi o de avaliar um protocolo anestésico a ser utilizado sob ventilação espontânea, o referido animal não foi utilizado para a avaliação das condições cirúrgicas obtidas e qualidade de recuperação. Em outro animal (peso de $21 \mathrm{~kg}$, idade de 24 meses) submetido a osteossíntese radial, nenhuma complicação foi observada.

\section{Discussão}

Após a aplicação de medicação pré-anestésica os animais apresentaram estado de tranqüilização bastante evidente, chegando a assumir decúbito lateral, quando não manipulados, demonstrando sobejamente dos efeitos sedativos da levomepromazina (Cailar et al., 1959). Os efeitos analgésicos da droga, citados na espécie humana 
Tabela 3 - Glicose, eletrólitos, hematócrito e hemoglobina de cães anestesiados com éter glicerol guaiacol, quetamina e fentanil $(n=6)$

\begin{tabular}{|c|c|c|c|c|c|}
\hline & 0 & 5 & $30^{\prime}$ & 60 & $75^{\prime}$ \\
\hline Glicose (mg/di) & $109.3 \pm 4.84$ & $117.7 \pm 12.9$ & $112.7 \pm 12.9$ & $117.5 \pm 23.64$ & $106.5 \pm 23.94$ \\
\hline Sódio (mmol/l) & $145.8 \pm 0.98$ & $146.2 \pm 2.32$ & $146.2 \pm 2.3$ & $146.3 \pm 1.21$ & $147 \pm 1.27$ \\
\hline Potássio $(\mathrm{mmol} / \mathrm{l})$ & $4.0 \pm 0.14$ & $3.8 \pm 0.21$ & $3.8 \pm 0.29$ & $-3.6 \pm 0.27^{*}$ & $3.5 \pm 0.35$ \\
\hline Cloro (mmol/l) & $114 \pm 3.1$ & $115 \pm 1.55$ & $115.3 \pm 1.37$ & $114 \pm 3.23$ & $115 \pm 4.47$ \\
\hline Cálcio $(\mathrm{mmol} / \mathrm{l})$ & $4.5 \pm 0.93$ & $4.7 \pm 0.94$ & $4.5 \pm 1.01$ & $4.4 \pm 1.01$ & $4.4 \pm 1.01$ \\
\hline $\begin{array}{l}\text { Cálcio não ionizado } \\
(\mathrm{mmd} / \mathrm{l})\end{array}$ & $4.2 \pm 0.9$ & $4.1 \pm 0.64$ & $4.3 \pm 0.75$ & $4.0 \pm 0.71$ & $4.0 \pm 0.82$ \\
\hline Anion residual & $11.7 \pm 5.82$ & $9.7 \pm 4.41$ & $9.7 \pm 5.16$ & $10.7 \pm 4.41$ & $11.3 \pm 4.5$ \\
\hline OSM (mOsm) & $286.3 \pm 2.42$ & $287.2 \pm 5.12$ & $287.3 \pm 4.23$ & $287.8 \pm 3.43$ & $288.2 \pm 3.06$ \\
\hline Hematócrito (\%) & $29.3 \pm 1.37$ & $31.5 \pm 12.19$ & $32.2 \pm 14.09$ & $34.2 \pm 12.09$ & $32.67 \pm 9.83$ \\
\hline Hemoglobina ( $g / d l)$ & $9.8 \pm 0.45$ & $10.5 \pm 4 / 03$ & $10.7 \pm 4.65$ & $11.4 \pm 4.1$ & $10.9 \pm 3.36$ \\
\hline
\end{tabular}

0 - Momento imediatamente antes da aplicação de MPA

75'- 15 min. após término da manutenção anestésica

(Paradis, 1962), não se fizeram evidentes, porque os animais se ressentiram da punção arterial femoral, conforme observado por outros autores. Dois animais (G1) mostraram sinais de excitação (ganidos, movimentos de morder e de cavar) por provável reação bifásica induzida pela droga em receptores dopaminérgicos centrais (Tobin e Ballard, 1979; Shar̃pe et al., 1995), sem que disso resultasse prejuízo em sua manipulação.

A bradicardia induzida pelo fentanil é provavelmente devida à estimulação de núcleos vagais medulares e ainda por bloqueio da atividade cronotrópica simpática (Patschke et al., 1977; Reitan et al., 1978). A bradicardia leva a uma hipotensão arterial sistólica, diastólica e média por redução do débito cardíaco (Patschke et al., 1977; Reitan et al., 1978). Tais ações nefastas não foram observadas, conforme outros estudos, com a associação de quetamina e fentanil em cães (Valadão e Castro, 1983); considerandose que nestes o EGG não causa depressão do sistema cardiovascular (Tavernor e Jones, 1970), presume-se que a estabilidade dos parâmetros cardiovasculares possa ser devida ao uso de quetamina que, isoladamente na espécie, provoca taquicardia e hipertensão (Haskins et al., 1985), à administração intravenosa lenta, que aparentemente minimiza os efeitos bradicardizantes atribuíveis ao fentanil (Liu et al., 1976) e ainda à tolerância aguda que muitas vezes se observa quando da administração de fentanil em bolus sucessivos (Eisele et al., 1975). Cita-se que a hipercapnia observada em cães sob ventilação espontânea anestesiados com halotano e fentanil pode contribuir para o surgimento de hipotensão (Nolan e Reid, 1991), já que em cães hipercapneicos $\left(\mathrm{PaCO}_{2}\right.$ maior que $45 \mathrm{~mm} \mathrm{Hg}$ ) o fentanil parece apresentar um leve efeito depressor da contratilidade miocárdica (Eisele et al., 1975). Entretanto, é sabido que o dióxido de carbono provoca estimulação simpática (Steffey et al., 1975), o que também pode ter contribuído para a estabilidade cardiovascular observada.
A depressão respiratória dose-dependente observada é, possivelmente, devida à ação do fentanil (Harper et al., 1976), sendo produzida pela queda do volume minuto por redução da freqüência respiratória (Krahwinkel et al., 1975; Harper et al., 1976), sem alteração significativa no volume corrente (Drummond, 1983), com concomitante acidose respiratória, hipóxia e hipercapnia, aumento do deficit de base (Krahwinkel et al., 1975), diminuição da resposta ventilatória à hipóxia e à hipercapnia (Harper et al., 1976) e diminuição da saturação de oxigênio na hemoglobina (Bailey et al., 1990). A hipóxia observada poderia ter sido contornada pela administração de oxigênio. A hipercapnia observada foi semelhante à produzida em cães sob anestesia geral com enflurano (Ilkiw et al., 1993). Dois animais apresentaram respiração ofegante com taquipnéia no momento zero, o que acarretou um desvio padrão elevado no mesmo momento para a freqüência respiratória e para o volume corrente e minuto.

A hipocalemia, provavelmente, deveu-se ao uso de glicose como diluente da associação anestésica ou à levomepromazina, que deslocariam o íon para o meio intracelular (Cailar et al., 1959; DiBartola, 1992).

A hipotermia pode ter sido produzida pelo fentanil e pela levomepromazina (Paradis, 1962; Bailey e Stanley, 1990), já que a quetamina causa ligeira elevação na temperatura corpórea quando usada isoladamente em cães (Haskins et al., 1985), e o EGG não a alteral (Pandey et al., 1973).

Outros autores, utilizando infusão contínua de EGG, quetamina e xilazina em cães, também relataram que a mesma produz melhores resultados em cães maiores (Mezerova et al., 1992). Observou-se o mesmo no presente estudo quando de manipulações de periósteo, realizadas sem complicações, em um animal de maior porte mas com produção de apnéia pelo aumento da velocida- 
de de infusão da associação anestésica em um cão de porte pequeno. Dada à enorme diferença interindividual no tocante ao peso corporal na espécie canina, o ajuste de velocidade de infusão intravenosa deveria ser realizado em função da área de superfície corporal, minimizando a ocorrência desta disparidade de respostas.

A intubação orotraqueal foi realizada sem a menor dificuldade, talvez pela ação do EGG, fato já observado em eqüinos (Roberts, 1968). O globo ocular foi mantido centralizado e em miose puntiforme, provavelmente por ação central e direta sobre a íris por parte do fentanil (Bailey e Stanley, 1990), mantendo-se os reflexos oculopalpebrais parcial ou totalmente abolidos.

Apenas o aspecto farmacodinâmico foi levado em consideração na determinação da velocidade de infusão das drogas utilizadas, visto que não foi possível medir os níveis plasmáticos das mesmas. Aparentemente, a velocidade de infusão destas foi adequada face ao estímulo doloroso, tendo em vista as condições cirúrgicas obtidas. O protolocolo de infusão foi determinado mediante trabaIhos anteriores em cães (Benson et al., 1985a; Ilkiw et al., 1993) cujos achados foram todavia corrigidos até que se obtivessem os valores utilizados mediante resultados de estudo piloto. Neste, foram utililizadas soluções com diferentes concentração de EGG, quetamina e fentanil, ajustando-se as mesmas até que fossem abolidas respostas nociceptivas com a menor alteração possível das variáveis paramétricas estudadas.

Ao contrário dos resultados de outros estudos utilizando fentanil não foi observada agressividade no período pós-operatório imediato, talvez pela ação tranqüilizante da levomepromazina (Cailar et al., 1959), promovendo recuperação suave e sem excitação (Tobin e Ballard, 1979). Também não se observou rigidez torácica que pode ser produzida por opióides (McLeish, 1977), provavelmente devido ao EGG que, como miorrelaxante diminuiria a incidência desse efeito indesejável, visto que em humanos o midazolam atenua a severidade da mesma (Neidhart et al., 1989). Não foi observada salivação que poderia ser imputada tanto ao fentanil quanto à quetamina (Sampaio e Bernis, 1973; Jacobsojn e Hartsfield, 1993), talvez pela ação da levomepromazina, que possui propriedades antisecretórias (Cailar et al., 1959). Conforme previamente observado (Sampaio e Bernis, 1973), metade dos animais defecou imediatamente após a indução, no decorrer da manutenção ou durante a recuperação anestésica, fato imputável ao fentanil, que induz hipermotilidade colônica seguida por período de hipomotilidade (Bardon e Ruckebush, 1985).

Cita-se a formação de trombos em cães após uma única aplicação de EGG (Vieira et al., 1995). Sua importância clínicà deve ser corretamente avaliada, visto que alterações semelhantes observadas em eqüinos não precluiram seu uso nesta espécie (Herschl et al., 1992).

\section{Conclusão}

O uso de EGG, quetamina e fentanil em cães caracterizou-se pela depressão respiratória diretamente proporcional à dose utilizada. A realização de procedimentos cirúrgicos mais dolorosos, como osteossínteses, foi viável com a utilização desta associação anestésica, mas o surgimento de apnéia é uma ocorrência a ser esperada, já que quanto maior a analgesia produzida pelo fentanil, maior a depressão cardiorrespiratória (Arndt et al., 1984). Tanto a indução quanto a recuperação anestésica foram isentas de excitação. As maiores desvantagens desta associação residem na necessidade de algum tipo de equipamento para realização de eventual assistência ventilatória, e nos efeitos deletérios do EGG no endotélio venoso dos cães (Krahwinkel et al., 1975; Vieira et al., 1995).

\section{Abstract \\ Anaesthesia in dogs guaiphenesin, ketamine and fentanyl using in continuous intravenous infusion}

Eleven mongrel dogs were premedicated with methotrimeprazine. Anaesthesia was induced and maintained by continuous intravenous infusion of a $5 \%$ glucose solution containing guaiphenesin, ketamine and fentanyl. Heart and respiratory rates, tidal and minute volume, systolic, mean and diastolic blood pressure, temperature, arterial blood gases, hemoglobin oxygen saturation, anion gap, serum osmolality, serum oxygen concentration, packed cell volume, hemoglobin, glucose and electrolytes were measured in six dogs. Analgesia was investigated usind a forceps in the skin. In the other five dogs heart and respiratory rates and response to surgical stimulus were investigated. Respiratory acidosis, hypoximia and hypoventilation were observed. Respiratory depression was proportional to the infusion rate, particulary when high infusion rates were used for bone repair.

Key words: guaiphenesin; ketamine; fentanyl; continuous intravenous infusion; dogs

\section{Agradecimentos}

À Janssen Farmacêutica por ter gentilmente cedido o Fentani ${ }^{\circledR}$ utilizado neste estudo.

\section{Referências bibliográficas}

ARNDT, J. O., MIKAT, M., PARASHER, C. Fentanyl's analgesic, respiratory and cardiovascular actions in relation to dose and plasma concentrations in unanesthetized dogs. Anesthesiology, v. 61, p. 355-361, 1984.

BAILEY, P. L., STANLEY, T. H. Narcotic Intravenous anesthetics. In: MILLER, R. D. (Ed). Anesthesia, 3rd ed., New York: Churchill Livingstone, 1990, 2v. V. 1, Cap. 10, p. 281-366.

BAILEY, P. L. et al. Frequent hypoxemia and apnea after sedation with midazolan and fentanyl. Anesthesiology, v. 73, p. 826830, 1990. 
BARDON, T., RUCKEBUSH, Y. Comparative effects of opiate agonists on proximal and distal colonic motility dogs. Eur. J. Pharmacol., v. 110, p. 329-334, 1985.

BENSON, G. J., THURMON, J.C., TRANQUILLI, W. J. SMITH, C. W. Cardiopulmonary effects of intravenous infusion of guaifenesin, ketamine and xylazine in dogs. Am. J. Vet. Res. v. 46, p. $1896-1898,1986 a$.

BENSON, G. J., THURMON, J. C., TRANQUILLI, W. J. Intravenous infusion of glyceryl guaiacolate, ketamine and xylazine in dogs: cardiopulmonary responses. Vet. Surg. v. 14, p. 71, 1985b. Abstract.

CAILAR, J., DECOURT, A., RIOUX, J. Intérêt de la lévomépromazine (7044 R.P.) en anesthésiologie. La Presse Medicale, v. 67, p. 1645-1646, 1959.

CURI, P.R. Análise de medidas repetidas em experimentos biológicosa. R. B. Estat., v. 41, p. 137-150, 1980.

DiBARTOLA, S. P., MORAIS, H. S. Disorders of PotassiumHypokalemia and Hyperkalemia. In: DiBARTOLA, S. P. (Ed). Fluid Therapy in Small Animal Practice. Philadelphia: W. B. Saunders, 1992, 720p., Cap. 2, p. 89-115.

DRUMMOND, G. B. Comparison of decreases in ventilation caused by enflurane and fentanyl during anaesthesia. Br. J. Anaesth. v. 55, p. 825-835, 1983.

DUNNETT, C. New tables for multile comparisions with a control. Biometrics., v. 20, p. 482-491, 1964.

EISELE, J. H., REITAN, J.A., TORTEN, M., MILLER, C. H. Myocardial sparing effect of fentanyl during halothane anaesthesia in dogs. Br. J. Anaesth., v. 47, p. 937-940, 1975.

HARPER, M. H., HICKEY, R. F., CROMWELL, T. H., LINWOOD, $S$. The magnitude and duration of respiratory depression produced by fentanyl and fentanyl plus droperidol in man. $J$. Pharmacol. Exp. Ther., v. 199, p. 464-468, 1976.

HASKINS, S. C., FERVER, T. B., PATZ, J. D. Ketamine in dogs. Am. J. Vet. Res., v. 36, p. 1855-1860, 1985.

HERSCHL, M. A., TRIM, C. A., MAHAFFEY, E. A. Effects of $5 \%$ and $10 \%$ guaifenesin infusion on equine vascular endothelium. Vet. Surg., v. 21, p. 494-497, 1992.

ILKIW, J. E., PASCOE, P. J., HASKINS, S. C., PATZ, J. D., JAFFE, $R$. The cardiovascular sparing effect of fentanyl and atropine, administered to enflurane anesthetized dogs. Can. J. Vet. Res., v. 57, p. 248-253, 1993.

JACOBSON, J. D., HARTSFIELD, S. M. Cardiorespiratory effects of intravenous bolus administration and infusion of ketaminemidazolam in dogs. Am. J. Vet. Res., v. 54, p. 1710-1714, 1993.

KRAHWINKEL, D. J., SAWYER, D. C., EYSTER, G. E., BENDER, G. Cardiopulmonary effects of fentanyl-droperidol, nitrous oxide and atropine sulfate in dogs. Am. J. Res., v. 36, p. 1211-1219, 1975.

LIU, W., BIDWAL, A. V., STANLEY, T. H., ISERN-AMARAL; J. Cardiovascular dynamics after large doses fo fentanyl and fentanyl plus $\mathrm{N}_{2} \mathrm{O}$ in th edog. Anesth. Analg., v. 55, p. 168172, 1976.

McLEISH, I. Skeletal muscle rigidity in a dog following fentanyl/ droperidol administration: a case report. Vet. Anesth., v. 4, p. 2-4, 1977.

MEZEROVA, J., NEMECEK, L., SNASIL, M. Continuous intravenous anesthesia in dogs using a combination of xylazine, ketamine and guaifenesin. Vet. Med. (Prague), v.
37, p. 341-347, 1992.

MILLER, D. R. Intravenous infusion anaesthesia and delivery devices. Can. J. Anaesth., v. 41, p. 639-652, 1994.

NEIDHART, P., BURGENER, M. C., SCHWIEGER, I., SUTER, $P$. M. Chest wall rigidity during fentanyl and midazolamfentanyl induction: ventilatory and haemodynamic effects. Acta Anesth. Scand., v. 33, p. 1-5, 1989.

NOLAN, A. M., REID, J. The use of intraoperative fentanyl in spontaneously breathing dogs undergoing orthopaedic surgery. J. Vet Anaesth. v. 18, p. 30-34, 1991.

PANDEY, S. K., PANDIT, R. K., PATEL, M. R., SINGH, B. N. Observations with glyceryl guaiacolate on dogs. JNKVVRes. J., v. 7, p. 265-268, 1973.

PARADIS, B. Analgesic and anaesthetic properties of levomepromazine (Nozinan®) (R. P. 7044). Can. Anaesth. Soc. J., v. 9, p. 153-160, 1962.

PATSHKE, D., BRUCKNER, J. B., EBERLEIN, H. J., TARNOW, J., WEYMAR, A. Effects consumption in man. Can. Anaesth. Soc. J., v. 26, p. 168-172. 1979.

RATAJCZAK, K; SKRZYPCZAK, P. Anesthesia in dog by continuous infusion. Med. Wet., v. 49, p. 248-250, 1993.

REITAN, J. A., STENGERT, K. B., WYMORE, M. L., MARTUCCI, R. W. Central vagal control of fentanyl induced bradycardia during halothane anesthesia. Anesth. Analg., v. 57, p. 3136, 1978.

ROBERTS, D. The role of glyceryl guaiacolate in a balanced equine anesthetic. Vet. Med. Small Anim. Clin., v. 63, p. 157162, 1968.

SAMPAIO, R. BERNIS, W. O. A neuroleptoanalgesia produzida pela associação droperidol-fentanil em cães. Arq. Esc. Vet. UFMG, v. 25, p. 251-257, 1973.

SHARPE, M.D., DOBKOWSKI, W. B., MURKIN, J. M., KLEIN, G., YEE, R. Propofol has no direct effect on sinoatrial node function or on normal atrioventricular and accessory pathway conduction in Wolff-Parkinson-White syndrome during alfentanil/midazolam anesthesia. Anesthesiology, v. 82, p. 88-895, 1995.

STEFFEY, E. P., GILLESPIE, J. R., BERRY, J. D., EGER, E. I., RHODE, E. A. Circulatory effects of halothane and halothanenitrous oxide anesthesia in thje dog: spontaneous ventilation. Am. J. Vet. Res., v. 36, p. 197-200, 1975.

TAVERNOR, W. D., JONES, E. W. Observation of the cardiovascular and respiratory effects of guaiacol glycerol ether in conscious and anaesthetized dogs. J. Small Anim. Pract., v. 11, p. 177-184, 1970.

TOBIN, T., BALLARD, S. Pharmacology review. The phenotiazine "tranquilizers", J. Equine Med. Surg., v. 3, p. 460-469, 1979.

VALADÃO, C. A. A., CASTRO, E. A. Associação do cloridrato de ketamina e citrato de fentanil - II parte. In: ENCONTRO DE PESQUISAS VETERINÁRIAS, 6, 1983, Jaboticabal. Anais... Jabotical: Faculdade de Ciências Agrárias e Veterinárias, 1983, p. 45-46.

VIEIRA, F. A. F., LAUFER, R., LUNA, S. P. L., BANDARRA, E. P., MASSONE, F., CASTRO, G. B. Effect of $5 \%$ guaiphenesin on venous endothelium of dogs. Enviado para publicação no Canadian Veterinary Journal em 1995.

WHITE, P. F. Use of continuous infusion versus intermitent bolus administration of fentanyl or ketamine during outpatient anesthesia. Anesthesiology, v. 59, p. 294-300, 1983. 